\title{
Gefährliche Doppelnatur von Budgets und ihre Überwindung
}

Wie lange sind Budgets in Ihrer Unternehmung aktuell? Sind sie schon nach einem, zwei oder drei Monaten wieder Makulatur? Kaum hat das Jahr begonnen, leiden in der heutigen, unvorhersehbaren Zeit bereits viele Firmen unter nutzlosen, veralteten Budgetdaten. Sie können sich glücklich schätzen, wenn Sie nicht dazu gehören!

Zahlreiche Managementsysteme bestehen aus jährlich ausgehandelten Zielen, Plänen und Ressourcen, welche auf den wohlbekannten, etablierten und weitverbreiteten Budgetierungsprozessen beru-

\section{Autoren

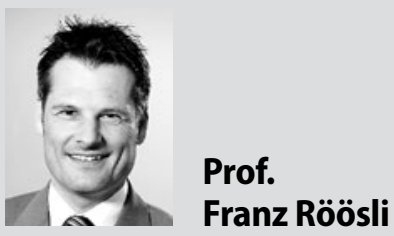

ist Dozent an der Fachhochschule Nordwestschweiz (FHNW), Management-Trainer und Director des Beyond Budgeting Round Table (BBRT), eine mitgliederbasierte Forschungs- und Praxiscommunity für Führung und Organisation. E-Mail: franz.roeoesli@fhnw.ch, www. fhnw.ch, www.bbrt.ch.

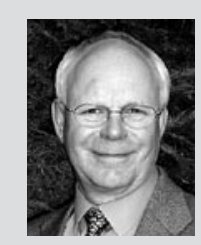

\section{Dr. Peter Bunce}

ist Diplomingenieur und hielt verschiedene Stellen in den Bereichen Fertigungstechnik und Zusammenarbeitsforschung. Er ist Mitbegründer des Beyond Budgeting Round Table (BBRT), eine mitgliederbasierte Forschungs- und Praxiscommunity für Führung und Organisation. E-Mail: peterbunce@bbrt. org, www.bbrt.org. hen. Diese Systeme wurden für ein stabiles Handlungsumfeld entworfen, in dem die Anbieter den Markt bestimmen konnten. Heutzutage sind viele Märkte bekanntlich stark umkämpft und die Kunden haben das Sagen (vgl. Hope 2006, S 3 f. u. S. 79 f.).

Dieser Artikel beleuchtet einerseits einen grundlegenden Konstruktionsfehler von Budgets. Andererseits beschäftigt sich der Artikel mit der Überwindung dieses Designfehlers und schlägt einen Weg vor, wie Organisationen vorausschauend neue Chancen ergreifen und sich an Risiken anpassen können, indem sie ein dynamisches, adaptives Rahmenkonzept von Zielen und Plänen verwenden, das auf einem rollierenden Forecastingzyklus beruht.

\section{Wer weiß am besten was läuft?}

Im heutigen schnelllebigen, volatilen Unternehmensumfeld sind die Teams im direkten Kontakt mit den Kunden am besten positioniert, um Veränderungen innerhalb der Märkte, unter den Mitbewerbern und bei Kunden wahrzunehmen und darauf zu reagieren. Sie müssen rasch auf sich abzeichnende Ereignisse antworten. Aus diesem Grund sollte diesen Teams die Verantwortung und Kompetenz gehören, um die nötigen Entscheidungen und Handlungen zu tätigen. Diese dynamische und hochgradig selbstorganisierende Art wertschöpfender Tätigkeit in Organisationen funktioniert nicht mit starren, jährlichen Planungszyklen, wie es die Budgetierung und die darauf beruhenden meist monatlichen Budget-Ist-Abweichungsanalysen verkörpern. Nötig ist ein Prozess simultanen und kontinuierlichen Vorausschauens und Handelns, in den stetig neues Wissen einfließt (vgl. Hope/Bunce/Röösli 2011, S. 209).

Mintzberg verwendet den Ausdruck, natürliche kontinuierliche Verbesserung (Englisch: Natural Continuous Improvement), um diesen Ansatz zu beschreiben. Er bezeichnet diesen Managementstil auch als ,ruhiges Management' (Englisch: Quiet Ma- nagement), bei dem es nicht darum geht, in der Business Class Champagner zu trinken, sondern darum, die Ärmel hochzukrempeln und herauszufinden was eigentlich gerade passiert. Dieser Ansatz wird nicht von oben aufgesetzt, sondern entwickelt sich von der Basis her (vgl. Mintzberg 1999, S. 29).

Mintzberg fängt damit die ganze Denkund Handlungsweise in entwicklungsfähigen, adaptiven Organisationen ein. In erster Linie geht es nicht um ausgeklügelte Werkzeuge, sondern um eine Einstellung und eine Denkweise. Solche Organisationen vertrauen ihren Mitgliedern, die über relevante und umsetzbare Informationen verfügen und verantwortlich handeln. Das Unternehmen baut auf sich selbstorganisierende Teams und tritt nicht wie ein Orchester auf, bei dem der Dirigent die Führung innehat und sämtliche Aktivitäten kontrolliert und steuert.

- Heutzutage sind Budgets schneller veraltet als die Zeit, die benötigt wird, um sie zu erstellen.

- Budgets haben einen Konstruktionsfehler: Sie verbinden Ziel und Plan zu einer Identität. Diese unnatürliche Doppelnatur von Budgets führt zu gravierenden Dysfunktionalitäten für Organisationen.

- Die Orientierungsleistung für Entscheidungen und Handlungen bei vorherrschender Dynamik kann nicht von Budgets erbracht werden, sondern vom aktuellen Wissen der Organisationsmitglieder, insbesondere jener mit Kundenkontakt.

- Lernende, adaptive Organisationen, welche sich in der heutigen Dynamik nachhaltig erfolgreich bewegen, bedienen sich vernetzter, flacher Strukturen mit einem rollierenden Forecastingzyklus. Der rollierende Forecastingzyklus eliminiert die Doppelnatur von Budgets und generiert kontinuierlich aktuelles Wissen, welches informierte Entscheidungen und Handlungen ermöglicht. 


\section{Freiheit und Ordnung widersprechen sich nicht}

Die meisten Wirtschaftsführer tun sich schwer mit der Gegebenheit, dass Organisationen keine zentrale Koordination und Kontrolle brauchen. Für Margaret Wheatley gilt jedoch folgende Erkenntnis: „Freiheit und Ordnung - zwei Kräfte, die wir in Gegensatz zueinander gestellt haben - stellen sich als Partner beim Erzeugen von gesunden und gut strukturierten Systemen heraus. Effektive Selbstorganisation wird durch zwei kritische Elemente unterstützt; einerseits durch einen klaren Sinn für Identität, andererseits durch Freiheit. Das gesamte System einer Organisation entwickelt mehr Zusammenhalt und Stärke, wenn die Mitglieder ihre eigenen freien Entscheidungen treffen und sich an einer klaren Organisationsidentität orientieren können. Die Organisation wird dabei weniger kontrollierend aber vernetzter und gleichzeitig ausgerichteter" (vgl. Wheatley 1999, S. 87). Anders gesagt: Effektive Koordination braucht keinen Koordinator. Koordination ist ein Teil des Systemdesigns und wird dem System nicht auferlegt. Fallbeispiele dafür sind dm-drogerie markt, W. L. Gore, Toyota, Svenska Handelsbanken, Egon Zehnder International, HCL oder Southwest Airlines. Der kalifornische Tomatenverarbeiter Morning Star ist ein weiteres überzeugendes Beispiel von Selbstorganisation, wie Gary Hamel im Januar 2012 beschrieben hat (vgl. Hamel 2012). Stark dezentralisierte, netzwerkartige Organisationen bieten große Vorteile bezüglich Lernfähigkeit, Anpassungsvermögen und Robustheit. Jene Art organisatorischen Designs legt den Grundstein für die Entfaltung des vollen Potenzials der Organisationsmitglieder. Dies ist etwas, das heutzutage dringend benötigt wird, um Lösungen zu finden, die sowohl die Kunden als auch die anderen Stakeholder einer Organisation zufriedenstellen. (vgl. Röösli/Sonntag 2011, S. 36).

Welche Art Managementprozesse wenden solche lernenden, adaptiven Organisationen an, um durch passende Zielsetzungen und Planungsmethoden stimmig unterstützt zu werden? Weiter unten werden wir aufzeigen, welche Managementprozesse in der immer turbulenteren Gegenwart und Zukunft nötig sind. Erst einmal möchten wir eine grundlegende Schwachstelle des herkömmlichen Budgetierens mit seinen daraus resultierenden Budgets aufzeigen, da wir das Verständnis jenes Designfehlers als wichtig erachten, um den grundlegenden
Unterschied unseres Lösungsvorschlages auf dieser Ausgangslage nachzuvollziehen.

\section{Die Doppelnatur von Budgets}

Budgets haben eine Doppelnatur, indem sie gleichzeitig ein Ziel und einen Plan darstellen. Diese Eigenart ist aus unserer Sicht ein Designfehler mit kontraproduktiven Effekten. Insbesondere schürt der Designfehler in immer dynamischeren Zeiten zunehmend Spannungen und schwerwiegende Fehlleistungen. So ist eine grundlegende Eigenschaft eines Ziels, dass es nicht gleichzeitig seinen eigenen Plan zu seiner Erreichung darstellen kann. Ein Plan ist vielmehr ein Mittel, ein Instrument, um ein Ziel zu erreichen, und nie das Ziel selbst eine schlüssige und üblicherweise unbestrittene Logik. Aber bei Budgets vollzieht man diese sinnwidrige Identität von Ziel und Plan, die einen gefährlichen Zirkelschluss verkörpert. Und dies ist fest eingraviert im verbreiteten und vorherrschenden Verständnis der Budgetierung mit den daraus resultierenden Budgets, welche einen so genannten ,fixierten Leistungsvertrag' darstellen (vgl. Hope/Bunce/Röösli 2011, S. 216f.). Das bedeutet nichts anderes als eine statische, im Voraus fixierte $\mathrm{Ab}$ machung in Form einer Zahl (Budget), der die dysfunktionale Ziel-Plan-Identität (Doppelnatur) innewohnt. Abbildung 1 stellt die problematische Doppelnatur von Budgets dar. Die Zielscheibe symbolisiert das Ziel, der Kompass den Plan.

Die Identität von Ziel und Plan in Form von Budgets ist im traditionellen Management als Axiom so tief verwurzelt, dass un- seres Erachtens die verschiedenen Konzepte zur Verbesserung der Budgetierung (z. B. ,Moderne Budgetierung') nicht systematisch bei dieser paradigmatischen Schwachstelle ansetzen. Deshalb verbleiben solche Ansätze bei der Symptombekämpfung und sind nicht als Wege zur ursächlichen Problemüberwindung einzustufen. Peter Ulrich, emeritierter Professor für Wirtschaftsethik der Universität St. Gallen, nennt das Phänomen des Erreichens geplanter Zahlen die ,bürokratische Zielverschiebung' (vgl. Puntas Bernet 2009, S. 47), bei welcher sich der Plan zum Ziel verschiebt respektive verrückt! Wir nennen das Phänomen den ,fixierten Leistungsvertrag. Nach unserer Meinung ist diese Doppelnatur Hauptursache der dysfunktionalen Effekte von Budgetierung und Budgets. Bekannte Beispiele für solche Dysfunktionalitäten sind langwierige Verhandlungsspiele bei der Budgetierung (häufig ,Gaming' genannt), die Orientierung an nicht mehr aktuellen Größen, sinnlose sowie zeitraubende und demotivierende Rechtfertigungsübungen bei Budget-Ist-Abweichungsanalysen, Verschiebungen von Zahlungsströmen in andere Abschlussperioden in Abhängigkeit der Budgeterreichung, unnötiges Ausschöpfen von Budgets zur Sicherstellung der Höhe von Folgebudgets etc. Und all dies zum Schaden der Organisation.

\section{Überlegungen im Hinblick auf eine Problemlösung}

Eine mögliche Lösung des zugrunde liegenden Problems muss systematisch bei der Trennung von Zielen und Plänen an-

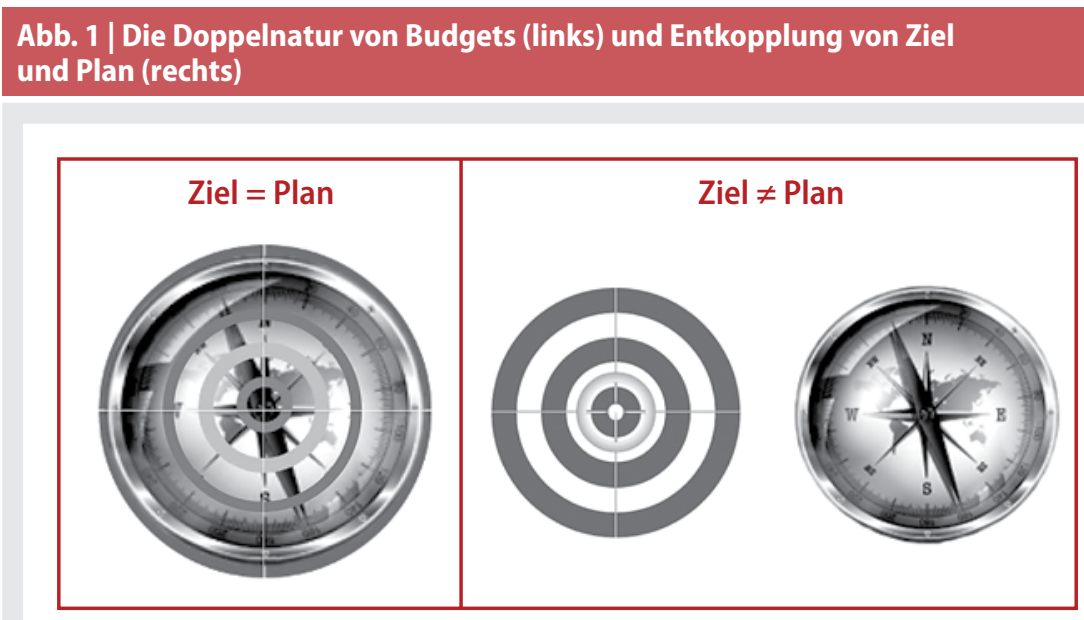


setzen. Die Auflösung der oben beschriebenen Doppelnatur, wie sie Budgets verkörpern, ist eine Voraussetzung, damit Entscheidungen und Handlungen von Organisationen mit der heutigen, sich schnell verändernden Welt Schritt halten können. Das bedeutet auch, dass die Illusion, mit fixierten Leistungsverträgen (Budgets) in dynamischen Zeiten die Kontrolle zu haben, aufgegeben werden muss (vgl. Hope/ Bunce/Röösli 2011, S. 7).

Die Budgetierung als operatives Steuerungsinstrument trat seinen Siegeszug in die Unternehmenspraxis vor rund hundert Jahren an, bei erheblich stabileren Umfeldbedingungen im Vergleich zur heutigen dominierenden hohen Dynamik. Insbesondere regierte ein sogenannter Verkäufermarkt bei dem die Produzenten an der Macht waren. Durch den Nachfrageüberhang konnten die produzierten Erzeugnisse praktisch ohne Lager verkauft werden. Die Verkaufsbudgets ergaben sich somit als direkte Folge aus der Budgetierung der Produktion. Die Produktionsplanung konnte aufgrund vor- handener maschineller Produktionskapazitäten deterministisch und relativ genau vorgenommen werden. In diesem Kontext erhielten die Budgets neben der Planfunktion auch Zielfunktionalität. Planerfüllung bedeutete folglich gleichfalls Zielerreichung (vgl. zur Budgetierung als zentrales Postulat im Industriezeitalter McKinsey 1922). In den statischen Zeiten von damals hat diese Ziel-Plan-Identität (Doppelnatur von Budgets) funktioniert. Aber nur im Sinne einer Ausnahme - nämlich unter der Bedingung von stabilen Umfeldfaktoren. Diese Bedingung existiert nicht mehr! Ein Steuerungsinstrument, das im Ausnahmefall stabiler Zeiten funktioniert hat, kann nicht ohne Dysfunktionalitäten bei der heute allgemein vorherrschenden Dynamik angewandt werden. Der damalige, über längere Zeit erfolgreiche Einsatz der Budgetierung mit Budgetzielen führte jedoch zum unreflektierten Einsatz dieses Steuerungsinstrumentes bis dato und ließ uns vergessen, dass die Bedingungen in der heutigen Zeit völlig anders aussehen.

\section{Abb. 2 | Der rollierende Forecastingzyklus und seine Einflussfaktoren}

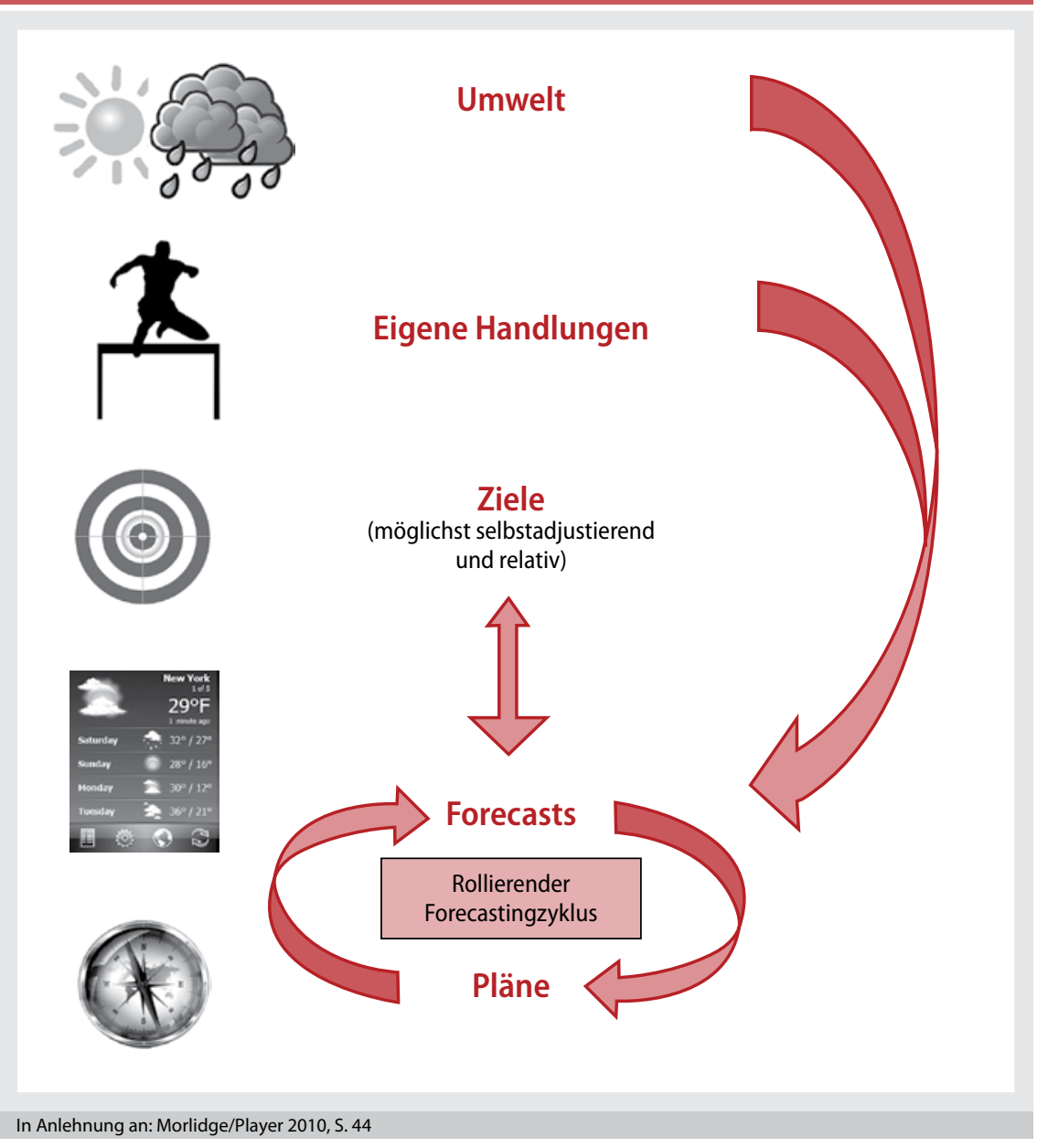

Der Leitgedanke für eine effektive Problemlösung unter Berücksichtigung heutiger dynamischer Umfeldfaktoren darf daher nicht Budget- oder Planerfüllung und deren Anreizung sein, sondern kontinuierliche Entwicklungs- und Anpassungsfähigkeit in Bezug auf Chancen und Gefahren aus dem Umfeld, z. B. bei Kunden, Mitbewerbern, Technologie, Gesetzgebung, etc. Es geht also um fortdauernde Innovation und Verbesserung um gegenüber dem Wettbewerb bestehen zu können und die Nase vorn zu haben. Die kollektive Intelligenz und das volle Potenzial der Organisationsmitglieder sind miteinzubeziehen. Entscheidungen und Handlungen werden dort ausgeführt, wo das größte Wissen um den Sachverhalt vorhanden ist und am schnellsten auf verändernde externe Bedingungen reagiert werden kann. Mit dieser Haltung und einer entsprechenden Systematik ohne fixierte Leistungsverträge kann verbreiteten Dysfunktionalitäten erfolgreich die Basis entzogen werden - wie beispielsweise absurden Verhandlungsspielen, demotivierenden Alibiübungen, absicherungsorientierter Ressourcenverschwendung und incentive-maximierender Zahlenschieberei.

\section{Rollierender Forecastingzyklus}

Aufgrund oben skizzierter Überlegungen schlagen wir einen rollierenden Forecastingzyklus als Lösung zur Überwindung der dysfunktionalen Doppelnatur von Budgets vor und möchten an dieser Stelle unsere hauptsächlichen Überlegungen dazu erläutern.

Was ist ein Forecast und wie unterscheidet er sich von einem Ziel oder einem Plan? Ein Forecast ist eine Prognose oder Projektion, die auf Annahmen über den zukünftigen Zustand der Realität basiert. Annahmen spielen eine Schlüsselrolle im Forecasting. Wir können über einen Forecast kein Urteil fällen, ohne dass wir die zugrunde liegenden Annahmen kennen. Ein häufiges Missverständnis ist, dass ein Forecast einer Prophezeiung gleichkommt - einer Wahrsage darüber, was passieren wird (vgl. Morlidge/ Player 2010, S. 39 ff.). Und genau dies trifft man vielfach in der Geschäftswelt, sogar beim Einsatz von rollierenden Forecastingkonzepten. Die neuen Daten werden zu verpflichtenden Erwartungen, welche somit zum Kontrollmaßstab werden. Das bedeutet im Umkehrschluss, dass diese neuen Forecasts fälschlicherweise zu Zielen kon- 
vertieren. Dadurch hat sich der Effekt der Doppelnatur von Budgets auch im rollierenden Forecastingkonzept eingeschlichen. Die Dysfunktionalitäten dieses Konstruktionsfehlers akzentuieren sich noch mehr durch die erhöhte Frequenz bei rollierenden Konzepten. Bürokratische Leerläufe und sinnloser Aktionismus sind beobachtbare Folgen.

Diese Problematik unterminiert geradewegs den Zweck, was wir mit Forecasts meinen, nämlich das Generieren von vorausschauendem Denken und Handeln in dynamischen Zeiten. Es geht bei Forecasts nicht um Wahrsagerei und die Kontrolle über zukünftige Resultate, denn die einzige Gewissheit in der heutigen turbulenten Zeit über Forecasts ist, dass sie falsch sein werden (vgl. Hope/Bunce/Röösli, S. 215). Planungssicherheit gibt es in dynamischen Umfeldern nicht - wer sich trotzdem so verhält, als gäbe es sie, vernichtet Wert für die Organisation. Nicht Wissen schützt an dieser Stelle vor Strafe nicht.

Ein Forecast ist somit eine Einschätzung über die zukünftige Realität. Die Ergebnisse eines Forecasts beziehen die Wirkungen von Annahmen über das externe, nicht direkt beeinflussbare Umfeld und die Wirkungen der eigenen, bereits beschlossenen Handlungen mit ein. Rollierende Forecastingzyklen sollten regelmäßig, beispielsweise monatlich oder quartalsweise, abhängig von der Marktdynamik der Branche und zudem ereignisorientiert initialisiert sowie durchgeführt werden. Ereignisorientiert bedeutet zum Beispiel eine sich plötzlich verändernde Kundenstruktur wird sichtbar, unerwartete Preisbewegungen tauchen auf, etc. Zudem decken rollierende Forecastingzyklen einen gleichbleibenden Zeithorizont in der Zukunft ab (z.B. jeweils die nächsten fünf Quartale). Man fährt also bei rollierenden Forecastingzyklen nicht gegen die Jahresendwand, als ob die Welt mit dem kommenden Jahr neu beginnt. Bildlich gesprochen bleiben die Scheinwerfer in die Zukunft gleichbleibend lang bei rollierenden Forecastingzyklen und werden nicht immer kürzer, je mehr man sich dem Geschäftsjahresende nähert. Ebenso bedeutsam ist es, das kollektive Wissen der gesamten Organisation systematisch in den Forecastingprozess einfließen zu lassen. Dies ist beispielsweise mit so genannten Informationsmärkten, unterstützt durch IT, realisierbar (vgl. Röösli/ Gebauer 2010, S. 106 f.).

Sollte sich ein Forecast ergeben, welcher darauf hinweist, dass die verfolgte Zielrichtung gefährdet ist oder sich neue
Chancen ergeben, sind Entscheidungen über mögliche zusätzliche Handlungen zu fällen, die ihrerseits sogleich im Forecastingzyklus integriert werden (beabsichtigte Handlungen sind Pläne - wir kommen im Artikelverlauf noch etwas detaillierter darauf zurück). Das führt wiederum zu einem neuen Forecast. Der aktuell erarbeitete Forecast kann sich beim nächsten Forecastingzyklus abermals aufgrund von neuen Erkenntnissen und zu initialisierenden Handlungen verändern. So geht es kontinuierlich mit Entscheidungen und Handlungen basierend auf periodischen und/oder ereignisorientierten Forecastingzyklen weiter. Damit ist der Zweck von rollierenden Forecastingzyklen, nicht die Zukunft vorherzusagen, sondern zu helfen, sie zu kreieren (vgl. Morlidge/Player 2010, S. 53). Abbildung 2 stellt den Forecastingzyklus im Kontext dar.

\section{Selbstadjustierende, relative Ziele}

Wie bereits erwähnt, sind Ziele und Forecasts nicht dasselbe. Im Gegensatz zu Forecasts, welche eine Einschätzung auf Basis heutigen Wissens über die Zukunft abgeben, beschreiben Ziele wie die Zukunft gemäß unseren Absichten aussehen sollte. Ziele sind demnach eine Willensbekundung. Lernfähige, adaptive Organisationen mit einer dezentralen Führung und Planung verwenden häufig selbstadjustierende, relative Ziele. Die Idee dahinter ist, dass sie relativ zum Wettbewerb gesetzt sind und nicht intern ausgehandelte oder vorgebebene Größen darstellen. Denn es ist für ein Unternehmen viel wichtiger, nachhaltig bessere Resultate als seine Mitbewerber zu erzielen, anstatt intern festgelegte Budgets zu erfüllen oder zu übertreffen. Ein Beispiel für ein relatives, selbstadjustierendes Ziel trifft man bei der schwedischen Bank Svenska Handelsbanken: Bei diesem Unternehmen ist die oberste finanzielle Zielsetzung eine höhere Eigenkapitalrendite zu erzielen als der Durchschnitt ihrer Mitbewerber. Eine solche wettbewerbsorientierte, dynamische Zielsetzung lässt sich nicht budgetieren, weil sie nicht intern aufgrund von Interessenlagen verhandelt ist, sondern die Mitbewerber als Relevanzmaßstab nimmt. Auch der norwegische Öl- und Gaskonzern Statoil wendet seit ein paar Jahren erfolgreich relative Ziele an (vgl. Bogsnes 2009, S. 119 ff.). Und genauso funktioniert es in vielen Sportarten, z. B. im Hochsprung. Beim Hochsprung gewinnt man nicht, wenn man plant, die Latte bei 2,40 Meter zu überspringen und dies sogar umsetzt, die Konkurrenz aber noch höher springt. Wenn man im Sport gewinnen will, muss man die Konkurrenz übertreffen und nicht einen selbst gesetzten Maßstab erreichen. Gewinner im Sport nehmen diese Herausforderung an.

\section{Plan als zukünftige Handlung}

Wenn also ein Forecast das ist, was unsere heutige Einschätzung über die Zukunft darstellt und ein Ziel das ist, was wir willentlich realisieren wollen - was ist dann ein Plan? Ein Plan ist ein Set von miteinander zusammenhängenden zukünftigen Handlungen, welcher entworfen wurde, um ein Ziel zu erreichen (vgl. Morlidge/ Player 2010, S. 42 f.). Pläne sind somit letztlich nichts anderes als angedachte Aktivitäten, die im Forecast einfließen, um zu beurteilen, ob man die richtige Richtung zur Unterstützung der Zielerreichung eingeschlagen hat. Pläne im Sinne dieses vorgeschlagenen Forecastingzyklus sind als zu initialisierende künftige Handlungen zu verstehen mit der Bewandtnis, mögliche Lücken zwischen der Einschätzung der Zukunft - ermittelt über Forecasts - und den gesetzten Zielen, die man erreichen will, zu schließen. Die Berücksichtigung dieser geplanten Aktivitäten führt wiederum zu entsprechenden Anpassungen beim Forecast. Dieses dynamische, iterative Momentum von Forecast, Planung von Aktivitäten und Anpassung von Forecasts setzt sich beim nächsten Forecastingzyklus kontinuierlich fort (vgl. zu diesen Ausführungen wiederum Abbildung 2). Der Sinn des Forecasts ist niemals seine Bestätigung sondern das fortwährende Generieren von Entscheidungen und geplanten Handlungen in einem dynamischen Umfeld zur Erreichung von relativen, wettbewerbsorientierten Zielen und damit zur Aufrechterhaltung der Wettbewerbsfähigkeit und Prosperität.

\section{Schlussfolgerungen}

Ein rollierender Forecastingzyklus, im oben beschriebenen Sinne, ist ein guter Weg für lernfähige, adaptive Unternehmen, vorwärts zu gehen, sich gegenüber den Mitbewerbern in der heutigen und künftigen immer turbulenteren Welt zu behaupten und 
$\mathrm{zu}$ prosperieren. Jedoch möchten wir abschließend mit Nachdruck darauf hinweisen, dass ein solches Forecastingsystem nur fruchtbar sein kann, wenn der gravierende Konstruktionsfehler der Doppelnatur von Budgets bei der Anwendung von Forecasts komplett vermieden wird. Daher darf ein Forecast nie einen Zielcharakter erhalten, dessen Erreichung kontrolliert wird und an welche eine Anreizung (Incentivierung) gekoppelt ist. Sobald eine Anreizung an die Erreichung einer Forecastgröße gekoppelt wird, wird diese Größe zum faktischen Ziel, mit den entsprechenden dysfunktionalen Auswirkungen, wie sie auch bei der Doppelnatur von Budgets einverleibt sind. Der Erfolg darf also nicht im Vergleich zu Forecasts beurteilt werden sondern im Vergleich $\mathrm{zu}$ den relativen, wettbewerbsorientierten Zielen. Hier schließt sich erneut der Vergleich zum Sport an, wo auch erst nach dem Wettkampf klar ist, wo man genau steht und ob man Lorbeeren ernten kann.

Wir laden Sie ein, über unsere vorgeschlagenen Ideen von Zielsetzung, Forecasting und Plänen kritisch nachzudenken und sich ebenso kritisch mit den momentanen Managementprozessen in Ihrer Organisation auseinanderzusetzen.

\section{Literatur}

1. Bogsnes, B.: Implementing Beyond Budgeting. Unlocking the Performance Potential, John Wiley \& Sons, Hoboken 2009.

2. Hamel, G.: Schafft die Manager ab! in: Harvard Business Manager, Heft Januar 2012, S. 22 - 36.

3. Hope, J.: Reinventing the CFO. How Financial Managers Can Transform Their Roles and Add Greater Value, Harvard Business School Press, Boston 2006.

4. Hope, J./Bunce, P./Röösli F.: The Leader's Dilemma. How to Build an Empowered and Adaptive Organization without Losing Control, Jossey-Bass, San Francisco 2011.

5. McKinsey, J.O.: Budgetary Control, Ronald Press, New York 1922.

6. Mintzberg, H.: Managing Quietly, in Leader to Leader, Edition Spring 1999, S. 24-30.

7. Morlidge, St./Player, St.: Future Ready. How to Master Business Forecasting, John Wiley \& Sons, Chichester 2010.

8. Puntas Bernet, D.: Abkehr von Kommando und Kontrolle, in: NZZ am Sonntag vom 18. Okt. 2009, S. 47.

9. Röösli, F./Gebauer, M.: Besser informieren, besser steuern, in: Harvard Business Manager, Heft April 2010, S. 106- 107.

10. Röösli, F./Sonntag, M.: Mehr Potenzial dank Vernetzungen, in: io management, Heft November/Dezember 2011, S. 34-37.

11. Wheatley, M.: Leadership and the New Science, Berret-Koehler Pulishers, San Francisco 1999.

\section{Alle Standards für das Studium} オ

springer-gabler.de

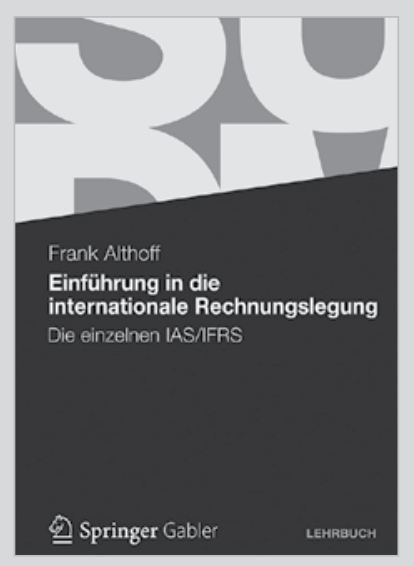

Frank Althoff

\section{Einführung in die \\ internationale Rechnungslegung}

Die einzelnen IAS/IFRS

2012. XX, 427 S. Br. € (D) 39,95

ISBN 978-3-8349-2999-0

Das Lehrbuch erläutert alle prüfungsrelevanten Standards und gibt mit zahlreichen kleinen Fällen einen Einblick in die Anwendung in der Praxis. Damit Sie für Studium und Beruf optimal vorbereitet sind.

\section{Springer Gabler}

Einfach bestellen: SpringerDE-service@springer.com Telefon +49 (0)6221/345- 4301 